\title{
Neurectomy versus Nerve Sparing in Open Inguinal Hernia Repair: A Randomised Controlled Trial
}

\author{
Shabbar Hussain Changazi, Nafeesah Fatimah, Asif Naseer, Abdul Wadood, Qamar Ashfaq Ahmad and \\ Mahmood Ayyaz \\ Department of General Surgery, Services Hospital, Lahore, Pakistan
}

\begin{abstract}
Objective: To determine the effect of neurectomy in reducing the frequency of chronic inguinodynia after mesh hernioplasty in open inguinal hernia repair.

Study Design: Single blind randomised controlled-trial.

Place and duration of study: Surgical Unit-I, Department of General Surgery, Services Hospital, Lahore, Pakistan from September 2018 to September 2019.

Methodology: All male patients undergoing open groin hernia surgery were included in the study. A total of 100 patients were randomly categorised into group A (neurectomy group) and group B (nerve sparing group). Patients were followed up for three months for the development of chronic inguinodynia. Signifiance was determined at $p<0.05$ using Chi-square and Fisher's exact tests.

Results: Out of 100 patients, 50 patients were enrolled in group A, while 50 were enrolled in group B. Mean age of patients was $42.1 \pm 17.5$ years. The median (IQR) acute pain score in neurectomy group was 3.0 (2.0-4.0), while median (IQR) acute pain score was 4.0 (3.0-6.0) in nerve sparing group with statistically significant difference $(z=-3.256, p=0.001)$. The frequency of chronic inguinodynia was significantly less in group A compared to group B [3 (6\%) vs. $13(26 \%), p=0.012$ ].

Conclusion: Excision of ilioinguinal and iliohypogastric nerve in inguinal mesh hernioplasty reduces the frequency of chronic inguinodynia. Ilioinguinal neurectomy may be practised routinely in patients undergoing Lichtenstein mesh hernioplasty.
\end{abstract}

Key Words: Inguinal neurectomy, Inguinal hernia, Chronic inguinodynia, Lichtenstein mesh hernioplasty.

How to cite this article: Changazi SH, Fatimah N, Naseer A, Wadood A, Ahmad QA, Ayyaz M. Neurectomy versus Nerve Sparing in Open Inguinal Hernia Repair: A Randomised Controlled Trial. J Coll Physicians Surg Pak 2020; 30(09):917-920 .

\section{INTRODUCTION}

Inguinal herniorrhaphy is one of the oft-done procedures in surgical practice. Around 20 million procedures are performed annually worldwide. ${ }^{1,2}$ Most hernia repairs are done without any serious complications. However, a few patients report postoperative pain and discomfort after inguinal hernia repair that influences the quality of life. ${ }^{3}$

Chronic inguinodynia is defined as pain persisting or arising at least three months following inguinal hernia repair after recovery from acute postoperative inflammation. The incidence of chronic groin pain ranges from $6.9 \%-60 \%{ }^{3,4}$ While acute pain can be successfully managed by analgesic therapy, chronic groin pain may become extremely difficult to manage ranging from non-operative to operative management. ${ }^{3,5}$

Correspondence to: Dr. Nafeesah Fatimah, Department of General Surgery, Services Hospital, Lahore, Pakistan

E-mail: nafeesahjavaid@gmail.com

Received: February 03, 2020; Revised: September 06, 2020; Accepted: September 30, 2020

DOI: https://doi.org/10.29271/jcpsp.2020.09.917
Hence, chronic groin pain can have significant psychological and social ramifications. ${ }^{1,4}$

Chronic groin pain usually results from damage to the ilioinguinal and iliohypogastric nerves. This manifests as pain and paresthesias in the distribution of these nerves. ${ }^{6}$ Inguinal neurectomy has been popularised as a treatment for incapacitating chronic inguinodynia after inguinal herniorrhaphy compared to other methods such as local infiltration of analgesics and laser therapy etc. ${ }^{3,7,8}$ Recent randomised controlled trials have shown lesser incidence of chronic inguinodynia after inguinal neurectomy compared to preservation of the nerves. In addition to randomised trials, various cohort studies have also shown lesser incidence of chronic groin pain after inguinal neurectomy. ${ }^{7-10}$

The objective of this study was to compare the effect of ilioinguinal and iliohypogastric neurectomy and nerve sparing practice in inguinal mesh hernioplasty in terms of chronic inguinodynia.

\section{METHODOLOGY}

This study was conducted in the Surgical Unit-I, Department of General Surgery, Services Institute of Medical Sciences, 
Lahore, Pakistan between September 2018 to September 2019. Ethical approval was sought from Institutional Review Board, Services Hospital, Lahore. This trial was registered in the international trials registry under the protocol ID NCT 03765268 (htt$\mathrm{p}: / / c$ linicaltrials.gov/). Written informed consent was obtained from the participants. All male patients aged between 16-75 years undergoing elective Lichtenstein mesh hernioplasty were included in the study by consecutive sampling. It was a single blind trial and patients were kept blind as to which treatment group they belonged to. Lottery method was used to randomise patients into two groups. Those patients with obstructed, strangulated, irreducible, bilateral inguinal hernia, recurrent inguinal hernia, uncontrolled diabetes mellitus, history of previous abdominal surgery and impaired cognition were excluded. Furthermore, patients who refused to give consent for open inguinal hernia surgery were also excluded. Lichtenstein tension-free mesh hernia repair was done under general or spinal anaesthesia, where appropriate. Trained general surgeons performed all surgeries.

The sample size was calculated considering a minimum $20 \%$ difference in the incidence of chronic inguinodynia, with the power of study $80 \%$, and level of significance of 0.05 . A total of 47 patients were required for each group. For cushioning purposes, the figure was rounded off to 50 .

The participants were randomly allocated by lottery method into group A and group B. Fifty papers marked group A and fifty papers marked group $B$, were placed in the box and patients included in the trial were asked to pick one paper. Hence, the group was allocated this way. In group A participants, ilioinguinal and iliohypogastric nerves were excised at the level of deep inguinal ring. In group B patients, nerves were spared and extreme caution was exercised not to incorporate the nerves in suture while anchoring the mesh. A standard size monofilament mesh was placed over the posterior wall of inguinal canal and anchored with the help of monofilament polypropylene sutures. All procedureswere performed by trained hernia surgeons. Postoperatively, all patients were managed with intravenous analgesia and scrotal elevation and were discharged within 24 hours.

The primary outcome measure was chronic groin pain, which was assessed on a follow-up visit 3 months after operation. Chronic inguinodynia was defined as any pain or discomfort reported by patients on a 3-month follow-up visit. Secondary outcome measure, i.e. acute postoperative pain was assessed by visual analog scale within 24 hours. To assure follow-up, the contact details of the patients were saved, and personalised reminders were sent to individual patients for scheduled follow-up.

Statistical analysis was performed with SPSS version 21 . Normality testing was done using Shapiro-Wilk test. Number and percentages were used for categorical variables while quantitative data were expressed as mean \pm standard deviation and median (interquartile range) where appropriate. Median pain scores were compared using Mann-Whitney U- test, while categorical data were compared using Fisher's exact test where appropriate. $P$ value $\leq 0.05$ was considered statistically significant. No life-threatening complications were noted during the trial; as a result, no modification was required in the conduction of the study.

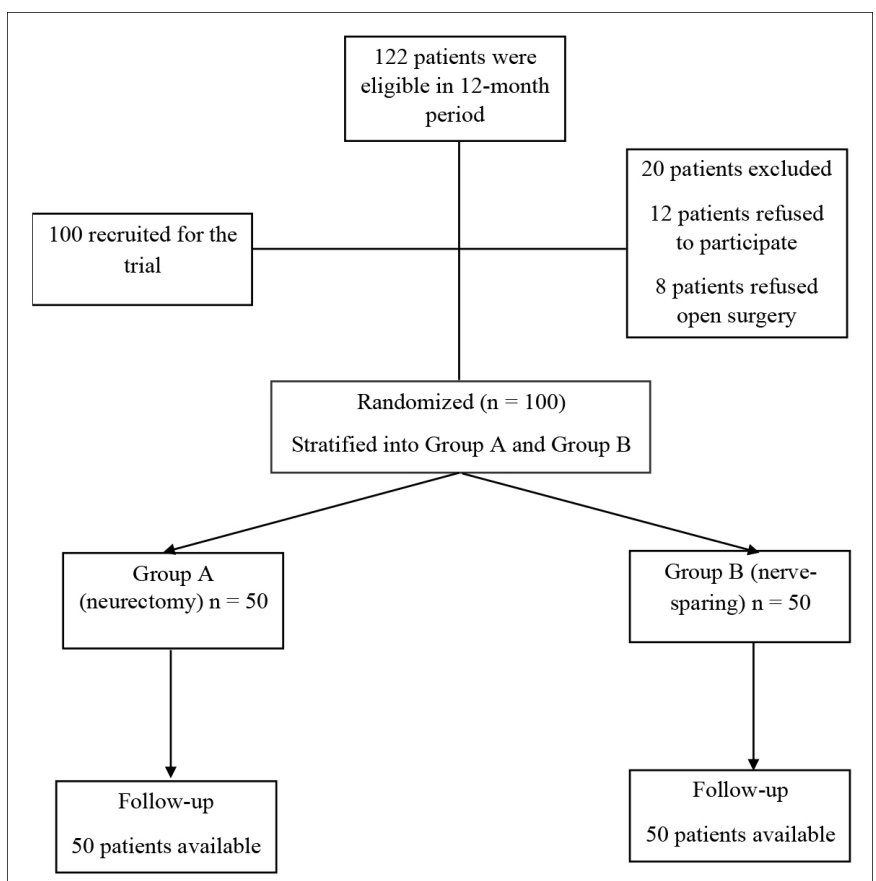

Figure 1: Randomisation tree diagram.

\section{RESULTS}

A total of 100 patients were recruited in the study during the one-year period. The mean age of patients was $42.1 \pm 17.5$ years. The mean age of patients was $40.2 \pm 17.0$ years in group A and $44.0 \pm 18.0$ years in group B. The two groups were compared with respect to age, mode of anaesthesia, laterality, and complications. The overall mean BMI of the patients was $25.1 \pm 3.2 \mathrm{Kg} / \mathrm{m}^{2}$ with mean BMl of $25.4 \pm 3.7 \mathrm{Kg} / \mathrm{m}^{2}$ in neurectomy group and $24.7 \pm 2.7 \mathrm{Kg} / \mathrm{m}^{2}$ in nerve sparing group.

Fifty (50\%) patients had incomplete inguinal hernia and 50 $(50 \%)$ had complete hernia. Out of 100 patients, 58 (58\%) patients had indirect inguinal hernia, while $42(42 \%)$ had direct inguinal hernia. Majority of the patients, i.e. $70 \%$ (70) had right sided hernia while 30\% (30) had left sided hernia.

Theiliohypogastric and ilioinguinal nerves were identified in all patients, which were excised in group A and carefully preserved in group $B$. The data was tested for normality using Shapiro-Wilk test ( $p=0.019)$, The median (interquartile range IQR) acute pain score in neurectomy group was 3.0, (2.0-4.0) while median acute pain score was 4.0 (3.0-6.0) in nerve sparing group with statistically significant difference $z=$ $-3.256, p=0.001$. All the 100 patients were available forfollowup after three months. The incidence of chronic inguinodynia was significantly lesser in group A compared to group B [3 (6\%) vs. $13(26 \%), p=0.012$ ]. 


\section{DISCUSSION}

Chronic inguinodynia is late and bothersome complication of inguinal mesh hernioplasty. ${ }^{1,2}$ It is known to significantly affect the quality of life causing psychological morbidity. ${ }^{5}$ Various mechanisms have been proposed as the cause of chronic groin pain ${ }^{11}$. One proposed mechanism is fibrosis around the ilioinguinal and iliohypogastric nerves due to placement of mesh. Moreover, accidental placement of suture on the nerves, while anchoring the mesh may also contribute to chronic groin pain. ${ }^{11,12}$ Multiple treatment modalities have been proposed ranging from simple analgesics to ilioinguinal neurectomy.

Neurectomy is being tested for the treatment of chronic inguinodynia. ${ }^{3,5}$ Hence, prophylactic neurectomy has recently been popularised for prevention of chronic inguinodynia and reduced numbness leading to decreased morbidity. ${ }^{2,5,6}$ The objective of our study was to compare the efficacy of prophylactic neurectomy versus nerve preservation in reducing chronic inguinodynia.

Various randomised controlled trials and retrospective studies have been done to study the result of ilioinguinal neurectomy on chronic inguinodynia and dysaesthesia. ${ }^{13-16}$ The Danish Hernia Register reports $29 \%$ incidence of chronic inguinodynia one year post-surgery with $11 \%$ of patients reporting severe pain; thus signifying the intensity of the problem and its long-term implications. ${ }^{13}$ The first study was done by Ravichandran et al. ${ }^{17}$ to compare the effect of neurectomy versus nerve preservation in chronic inguinodynia. It was done by randomising patients with bilateral inguinal hernias, i.e. doing neurectomy on one side and nerve-preservation on the other side. The study, despite being unable to show any significant difference between the two groups, paved way for further larger prospective studies. One reason could be the small sample size of the study $(\mathrm{N}=20)$.

One study done on 180 patients in Pakistan reported significant reduction in the incidence of chronic inguinodynia after neurectomy at one- and six-month follow-up ( $p<0.001$, respectively). ${ }^{8}$ Another study by Basimbe et al. showed a significant decline in the incidence of groin pain in the neurectomy group at one month of operation. ${ }^{18}$ However, no significant difference was seen six months after surgery. Various meta-analysis and systematic reviews have been done on this subject. A latest meta-analysis done by Barazanchi et al. showed decreased incidence of groin pain in short (half to $<3$ months) and mid-term ( 3 to $<12$ months); but no difference could be elicited on long-term basis (>12 months). ${ }^{19}$ Another meta-analysis reported similar findings in patients who had elective neurectomy compared to the control groups. However, the study reported increased incidence of paresthesia and numbness in the groin in neurectomy group.

Despite the increasing evidence of decreased groin pain after prophylactic neurectomy, there are still some studies with variable results. ${ }^{3,10}$ Another study done in India also showed decreased incidence in groin pain at six monthly follow-up at rest and after mild physical activity. ${ }^{10} \mathrm{~A}$ similar trial done in China looked at the development of chronic inguinodynia both at rest and after different levels of exertion, and found comparable results of chronic groin pain at rest in the two groups. ${ }^{3}$ However, they found that significantly fewer number of patients complaint of chronic groin pain after exertion (cycling for ten minutes and walking up three flights of stairs) in the neurectomy group and overall. Irrespective of the level of exertion, the incidence of chronic inguinodynia was lesser in the neurectomy group compared to the controls ( $8 \%$ vs. $28.6 \%)^{3}$

This randomised study was a short follow-up study. The median acute pain scores were higher in nerve preservation group compared to the neurectomy group. This difference was found to be statistically significant. Moreover, significantly lesser number of patients in this study developed groin pain in neurectomy group compared to the nerve preservation group (3 vs. 13). These findings further demonstrated that prophylactic ilioinguinal neurectomy had reduced chronic inguinodynia after Lichtenstein mesh hernioplasty; thus it can be routinely incorporated in inguinal hernia repairs. Due to its presence in the operative field, it is prone to damage. If during mesh anchorage, the nerve is trapped in one the anchoring sutures, groin pain and numbness mayensue.

The present study has many limitations. Pain was evaluated subjectively and it can be one of the biggest limitations as pain is a subjective feeling. The authors evaluated chronic groin pain irrespective of the level of exertion. The long-term effects of ilioinguinal nerve excision were not studied. Longer follow-up studies with more number of patients need to be done to better compare effect of neurectomy on chronic inguinodynia. There is a possibility that differences in the incidence of chronic groin pain might change with longer follow-up. Moreover, effect of neurectomy on quality of life was not studied as done in previous studies. Although we have been ableto show significant differences in the incidence of chronic groin pain between the two groups, the exact mechanism behind this is unknown. Histological and nerve conduction studies may provideanswer to this question.

\section{CONCLUSION}

This study redemonstrates that ilioinguinal and iliohypogastric neurectomy lessens the incidence of chronic inguinodynia. llioinguinal nerve excision can be practised as a routine surgical procedure in patients undergoing Lichtenstein mesh hernioplasty. However, longer follow-up studies with more number of participants would provide better solutions.

\section{ETHICALAPPROVAL}

Ethical approvals were received from all Institutional Review Board, Services Hospital, Lahore, Pakistan.

\section{PATIENTS' CONSENT}

Written informed consents were obtained from all patients before enrolling them in the study.

\section{CONFLICT OF INTEREST}

Authors declared no conflict of interest. 


\section{AUTHORS' CONTRIBUTION}

SHC, NF: Was involved in study design, data collection, data analysis, paperwriting and final approval of the manuscript.

AN: Involved in study design, data analysis, paper writing and final approval of the manuscript.

AW: Involved in study design, data collection, paper writing and final approval of the manuscript.

QA: Involved in study design, paper writing, and final review of the manuscript.

MA: Supervised the whole process from study design to final approval of the manuscript.

\section{REFERENCES}

1. Graham D, MacQueen I, Chen D. Inguinal neuroanatomy: Implications for prevention of chronic postinguinal hernia pain. Int J Abdo Wall Hernia Surg 2018; 1(1):1-8. DOI: 10.4103/ijawhs.ijawhs_6_18

2. Bjurstrom MF, Nicol AL, Amid PK, Chen DC. Pain control following inguinal herniorrhaphy: Current perspectives. J Pain Res 2014; 7:277-90. doi: 10.2147/JPR.S47005.

3. Lange JFM, Kaufmann R, Wijsmuller AR, Pierie JPEN, Ploeg $\mathrm{RJ}$, Chen DC, et al. An international consensus algorithm for management of chronic postoperative inguinal pain. Hernia 2015; 19(1): 33-43. doi: 10.1007/s10029-014-1292-y.

4. Bande D, Moltó L, Pereira JA. Chronic pain after groin hernia repair: pain characteristics and impact on quality of life. BMC Surg 2020; 20:147. doi.org/10.1186/s12893020-00805-9

5. Campanelli G, Bertocchi V, Cavalli M, Bombini G, Biondi A, Tentorio $T$, et al. Surgical treatment of chronic pain after inguinal hernia repair. Hernia 17:347-53. doi: 10.1007/ s10029-013-1059-x.

6. Andresen K, Rosenberg J. Management of chronic pain after hernia repair. J Pain Res 2018; 11: 675-681 doi: 10.2147/ JPR.S127820

7. Omar A, Rageh M, Kahter ZM. Effect of neurectomy versus ilioinguinal nerve preservation in the Lichtenstein tension-free hernioplasty of inguinal hernia. Menoufia Med J 2017; 31:152-157. doi: 10.4103/mmj.mmj_606_16.

8. Sharif A, Akhtar T, Akhtar M, Malik I, Hanif M, Zia N. Effect of prophylactic ilioinguinal neurectomy on postoperative groin pain following lichenstein hernioplasty. J Coll Physicians Surg Pak 2019; 29(5):406-9. doi: 10.29271/ jcpsp.2019.05.406.

9. Khoshmohabat H, Panahi F, Alvandi AA, Mehrvarz S, Mohebi $H A$, Shams Koushki E. Effect of Ilioinguinal neurectomy on chronic pain following herniorrhaphy. Trauma Mon 2012; 17(3):323-8. doi: 10.5812/traumamon.6581.

10. Sunkar S, Brashier BSD, Bhagwat K, Sharma V, Angrish P. A clinical study of the incidence and disability caused by mesh neuralgia after inguinal mesh hernioplasty and the effects of prophylactic ilioinguinal neurectomy and its role in preventing chronic groin pain after inguinal hernioplasty. Sunkar 2018; 5(3):3. doi: http://dx.doi.org/10.18203/23492902.isj20180835

11. Andresen K, Rosenberg J. Management of chronic pain after hernia repair. J Pain Res 2018; 11:675-81. doi: 10.2147/ JPR.S127820.

12. Heise $C P$, Starling JR. Mesh inguinodynia: A new clinical syndrome after inguinal herniorrhaphy? J Am Coll Surg 1998; 187(5):514-8. doi: 10.1016/s1072-7515(98)00215-4.

13. Gangopadhyay N, Pothula A, Yao A, Geraghty PJ, Mackinnon SE. Retroperitoneal approach for ilioinguinal, iliohypogastric, and genitofemoral neurectomies in the treatment of refractory groin pain after inguinal hernia repair. Ann Plast Surg 2020; 84(4):431-5. doi: 10.1097/SAP.0000000000002226.

14. Bande D, Molto L, Pereira JA, Montes A. Chronic pain after groin hernia repair: Pain characteristics and impact on quality of life. BMC Surg 2020; 20:147. http://doi.org/10. 1186/s12893-020-00805-9.

15. Charalambous MP, Charalambous CP. Incidence of chronic groin pain following open mesh inguinal hernia repair, and effect of elective division of the ilioinguinal nerve: Metaanalysis of randomized controlled trials. Hernia 2018; 22(3):401-409. doi: 10.1007/s10029-018-1753-9.

16. Vijayakumar A, Shivashankar S, Manangi M. Chronic pain after inguinal hernia repair. Int Sch Res Notices 2014; 2014:839681. doi: 10.1155/2014/839681.

17. Ravichandran D, Kalambe BG, Pain JA, 2000. Pilot randomized controlled study of preservation or division of ilioinguinal nerve in open mesh repair of inguinal hernia. $\mathrm{Br}$ J Surg 87(9):1166-7. doi: 10.1046/j.1365-2168.2000. 01529.x.

18. Basimbe F, Ongom PA, Kijjambu SC, Kituuka O. Postoperative pain in lichtenstein repair with lliohypogastric neurectomy compared to standard lichtenstein repair, for inguinal hernias at Mulago Hospital, A Sub Saharan Africa Tertiary Centre. J Gen Pract 2013; 1:128.

19. Barazanchi AW, Fagan PV, Smith BB, Hill AG. Routine neurectomy of inguinal nerves during open onlay mesh hernia repair: A meta-analysis of randomised trials. Ann Surg 2016; 264(1):64-72. doi: 10.1097/SLA.000000 0000001613. 